\title{
ANATOMIC VARIANTS AND ARTEFACTS IN NON ENHANCED MRV - POTENTIAL PITFALLS IN DIAGNOSING CEREBRAL VENOUS SINUS THROMBOSIS (CVST)
}

\author{
Pallewatte A S, Tharmalingam T, Liyanage N
}

Neuroimaging Unit, The National Hospital of Sri Lanka, Colombo, Sri Lanka

Keywords: MRV, cerebral venous sinuses, thrombosis, normal variants, artifacts, pitfalls

Correspondence: Pallewatte A S <asp31263@hotmail.com>

Copyright: Pallewatte A S

\section{INTRODUCTION}

Cerebral venous thrombosis is a neurological emergency reversible with specific treatment. Prompt diagnosis in a compatible clinical setting is the most important factor in proper management. Radiological imaging plays a major role in confirming the diagnosis and identifying associated complications. It also helps to differentiate other conditions which have similar clinical presentation.

Though there are many imaging modalities which may play a role in diagnosis of cerebral venous thrombosis such as non-contrast computed tomography (NCCT) brain, Computed Tomographic Venography (CTV) etc, Magnetic Resonance Imaging (MR) with Magnetic Resonance Venography (MRV) is the preferred diagnostic modality. The main advantages include the ability to perform MRV without any special patient preparation or intravenous contrast medium administration and the ability to assess the brain parenchyma simultaneously.

The two techniques used to image the venous anatomy in MR venography are time-offlight (TOF) and phase contrast (PC) techniques. TOF depends on the excitation of blood in one slice of the body and detecting it in another. It differentiates moving blood from the stationary tissues. The penetration of the excited blood into a imaged slice depends on the T1, velocity and direction of the flow.

Phase contrast depends on the phase change of moving protons in the blood. The data from moving protons is enhanced by 
subtracting data acquired twice using positive and negative gradients.

\section{NORMAL ANATOMY}

There are two groups of cerebral venous sinuses, superior group and the basal group. The sagittal, transverse and straight sinuses are the main parts of superior group, whereas the basal group consists of carvenous, petrosal and sphenoparietal sinuses.

The superficial cerebral veins flow in a centrifugal direction and drain in to the venous sinuses. There are three named superficial cerebral veins which are superficial middle cerebral vein of Sylvian, Veins of Trolard and Labbe. The last two connect the superficial middle cerebral vein with superior sagittal and transverse sinuses respectively.

The two deep cerebral veins, the thalamostriate and the septal vein join to form the internal cerebral vein behind the foramen of Monro. The basal vein of Rosenthal is formed in the Sylvian fissure. The paired internal cerebral veins and veins of Rosenthal join to form the single, short

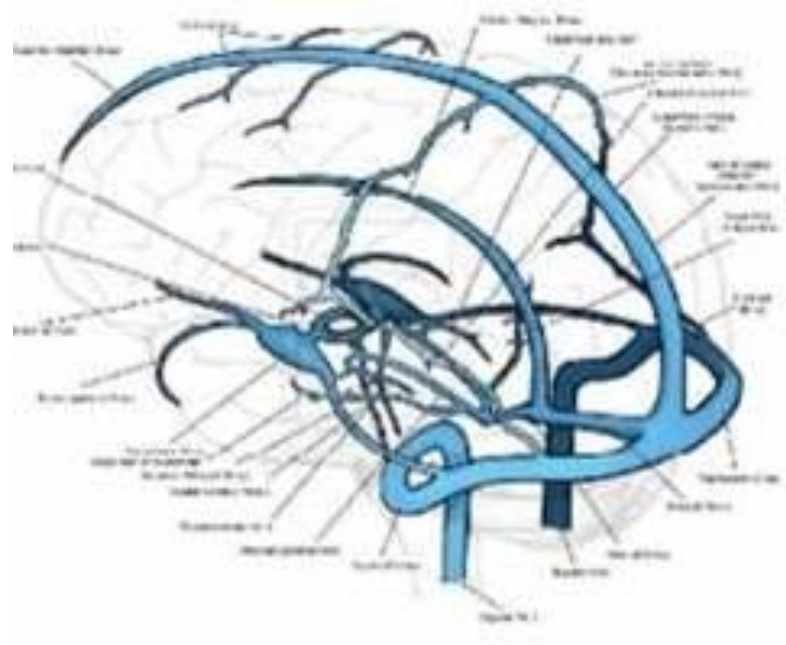

middle vein called great cerebral vein (of Galen) which in turn joins with inferior sagittal sinus to form the straight sinus. The straight sinus usually drains in to the left transverse sinus.

The superior sagittal sinus lies along the upper border of the falx cerebri, drains usually into the right transverse sinus. The transverse sinuses start at internal occipital protuberance and lie within the outer margins of the tentorium. The right is usually dominant and larger than the left. The transverse sinuses become sigmoid sinuses at the petrous edge and continue towards the jugular foramen, and joined by the superior petrosal sinus to form the internal jugular vein. Paired carvenous sinuses are situated on either side of the pituitary fossa and have inter carvenous sinus connections between them. Anteriorly they receive superior, inferior ophthalmic veins and sphenoparietal sinus. Posteriorly they drain in to the superior and inferior petrosal sinuses. Inferior petrosal sinuses drain in to the jugular bulb (Figure 1).

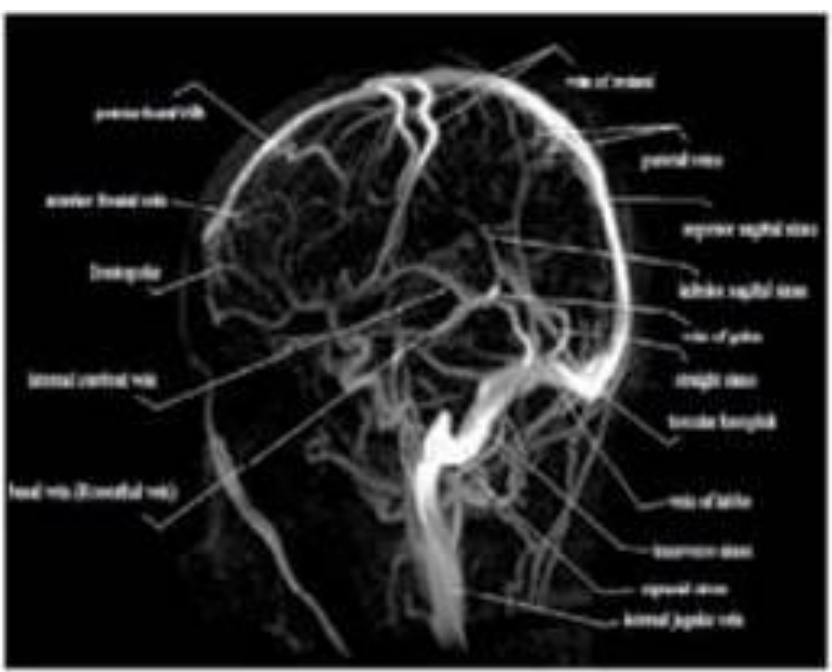

Figure 1. Normal cerebral venous anatomy as seen on a MR venogram 


\section{Pictorial essay $\mid$ SLJR}

\section{NORMAL VARIANTS}

Normal variations in venous anatomy are common on MR Venography. The following are frequently encountered. The incidence varies in literature. Approximate percentage denoted under each category represent the incidence found on study done at the authors' Institution.

\section{Dominance of transverse sinus}

The superior sagittal sinus usually drains in to the right transverse sinus. The transverse sinus which receives superior sagittal sinus is considered dominant. The dominant transverse sinus being right (58\%), left (27\%) of cases. Co dominance is seen in $15 \%$ (1) (Figure 2). sinus while only $10 \%$ had right side asymmetry (3) (Figure 3, 4).

\section{Hypoplasia of superior sagittal sinus}

Superior sagittal sinus is always present. There is hypoplasia noted in $5.7 \%$ in which anterior $1 / 4$ is hypoplastic in $3.3 \%$ cases and anterior $1 / 2$ is hypoplastic in $2.4 \%$ of cases (1) (Figure 5).

\section{Oblique sinus}

Oblique sinus is rare and acts as an alternative drainage pathway when transverse sinus is absent or hypoplastic. It was observed in 10\% of cases (4) (Figure 6).

Technical artifacts on MR Venography occur due to misregistration data and during
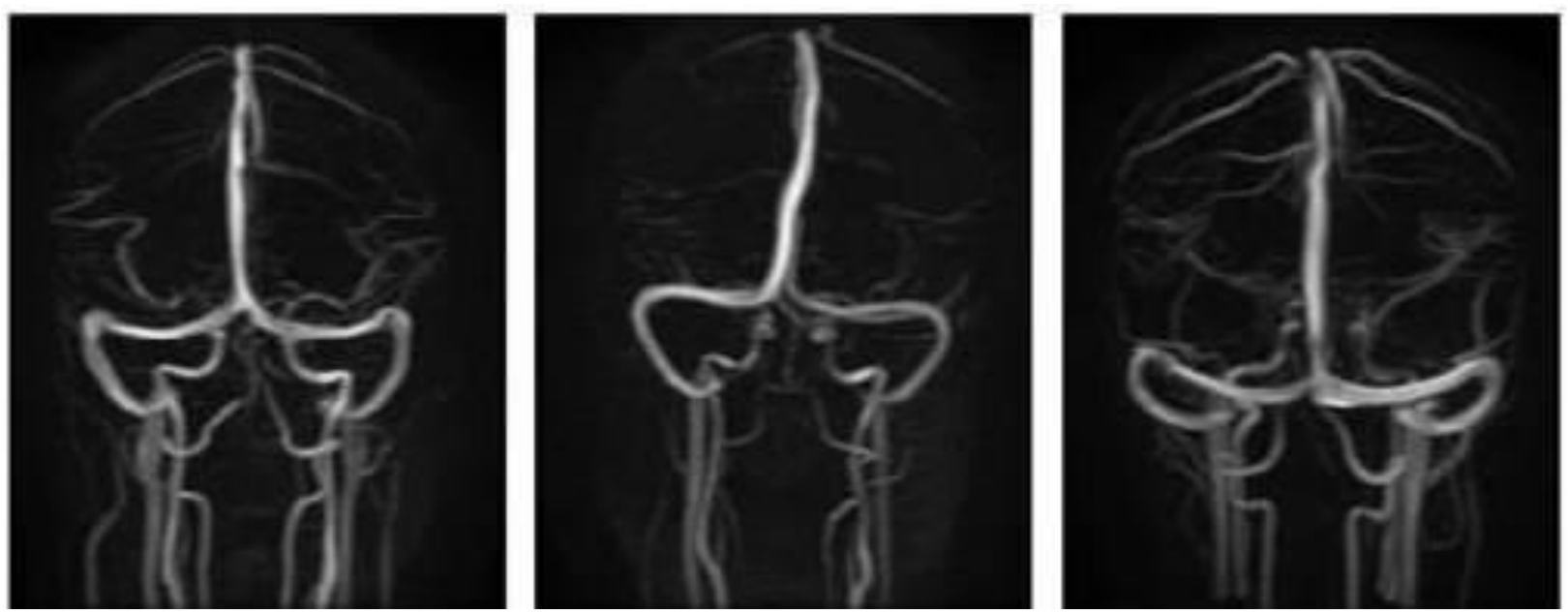

Figure 2. Co dominance, right and left dominance of transverse sinus

\section{Hypoplasia and atresia of transverse sinuses}

Hypoplasia and atresia of transverse sinuses occur frequently. Asymmetrical transverse sinuses seen in $49 \%$ of cases, while the partial or complete absence of one of the transverse sinus is seen in $20 \%$ cases (2) According to literature, asymmetry is usually observed on the left side. $59 \%$ of cases had either aplastic $(20 \%)$ or hypoplastic (39\%) left transverse reconstruction. These are also frequently seen in clinical MR imaging. Flow gaps in transverse sinuses /Flow gaps can be misdiagnosed as cerebral venous thrombosis.

\section{Flow gaps in transverse sinuses}

Flow gaps can be misdiagnosed as cerebral venous thrombosis. Flow gaps in transverse sinuses were observed in $31 \%$ of cases in transverse sinus (4) (Figure 7). 


\section{Pictorial essay $\mid$ SLJR}
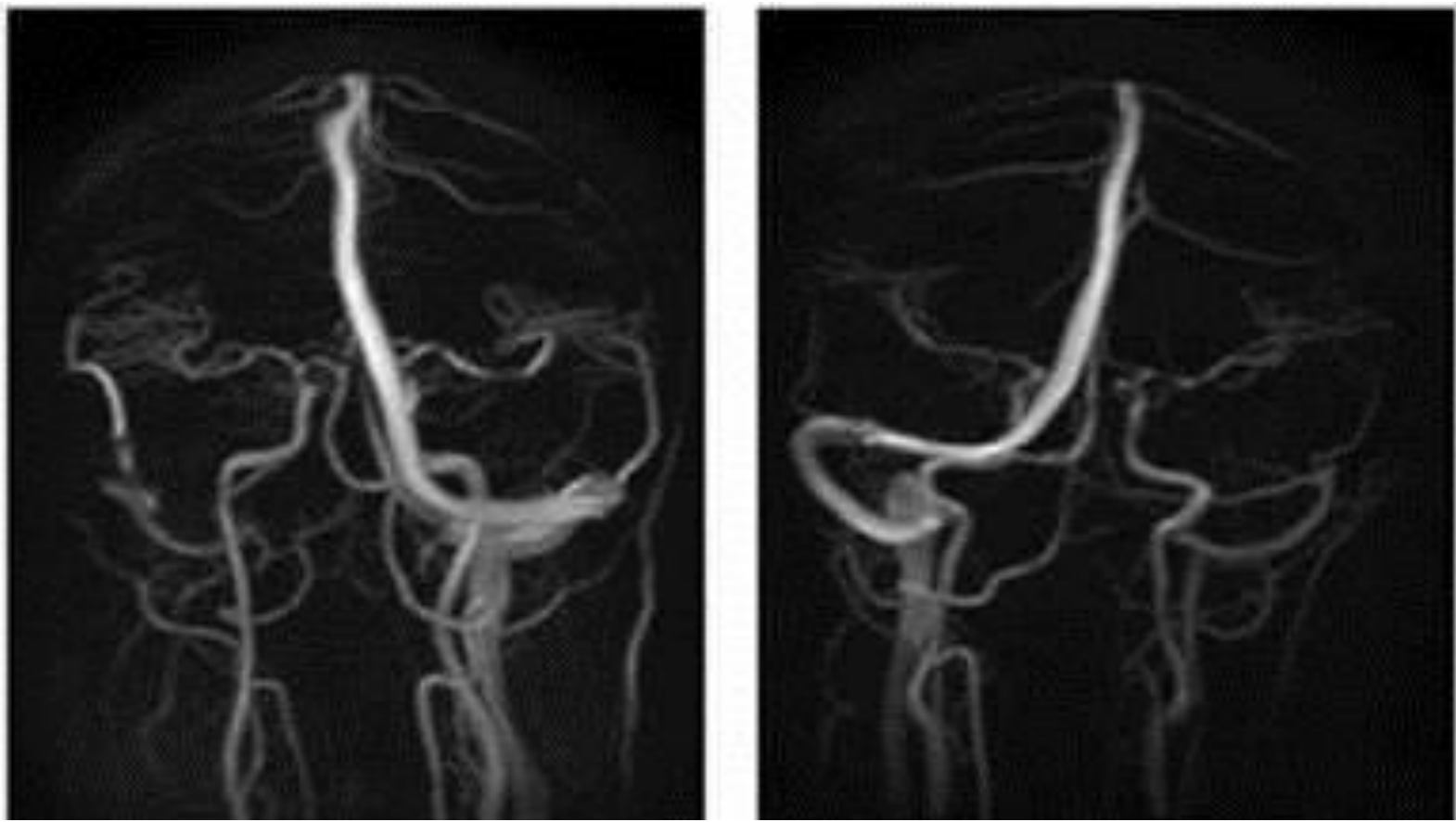

Figure 3. Atretic right and left transverse sinuses
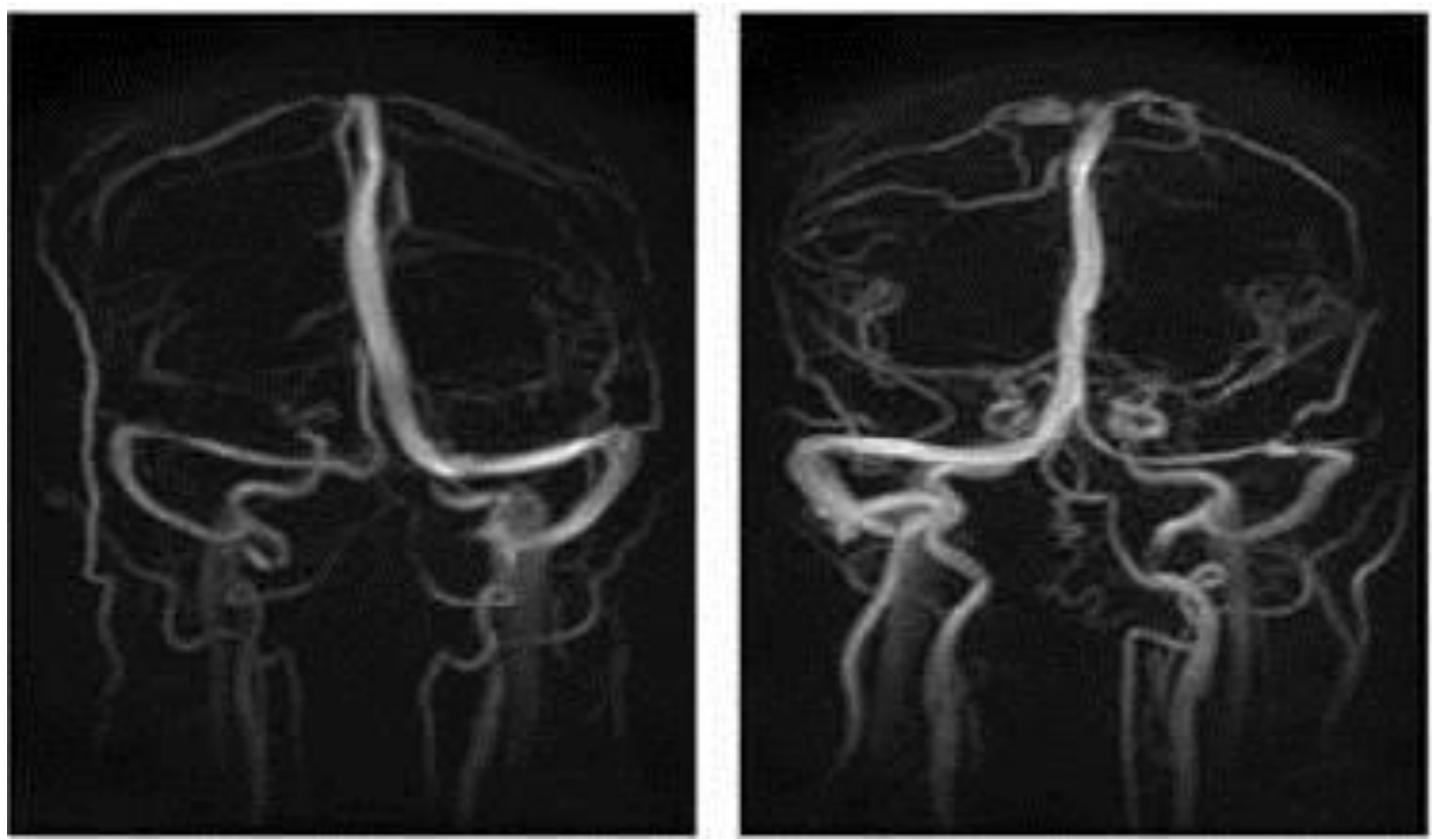

Figure 4. Hypoplastic right and left transverse sinuses MRV 


\section{Pictorial essay $\mathbf{S L J R}$}
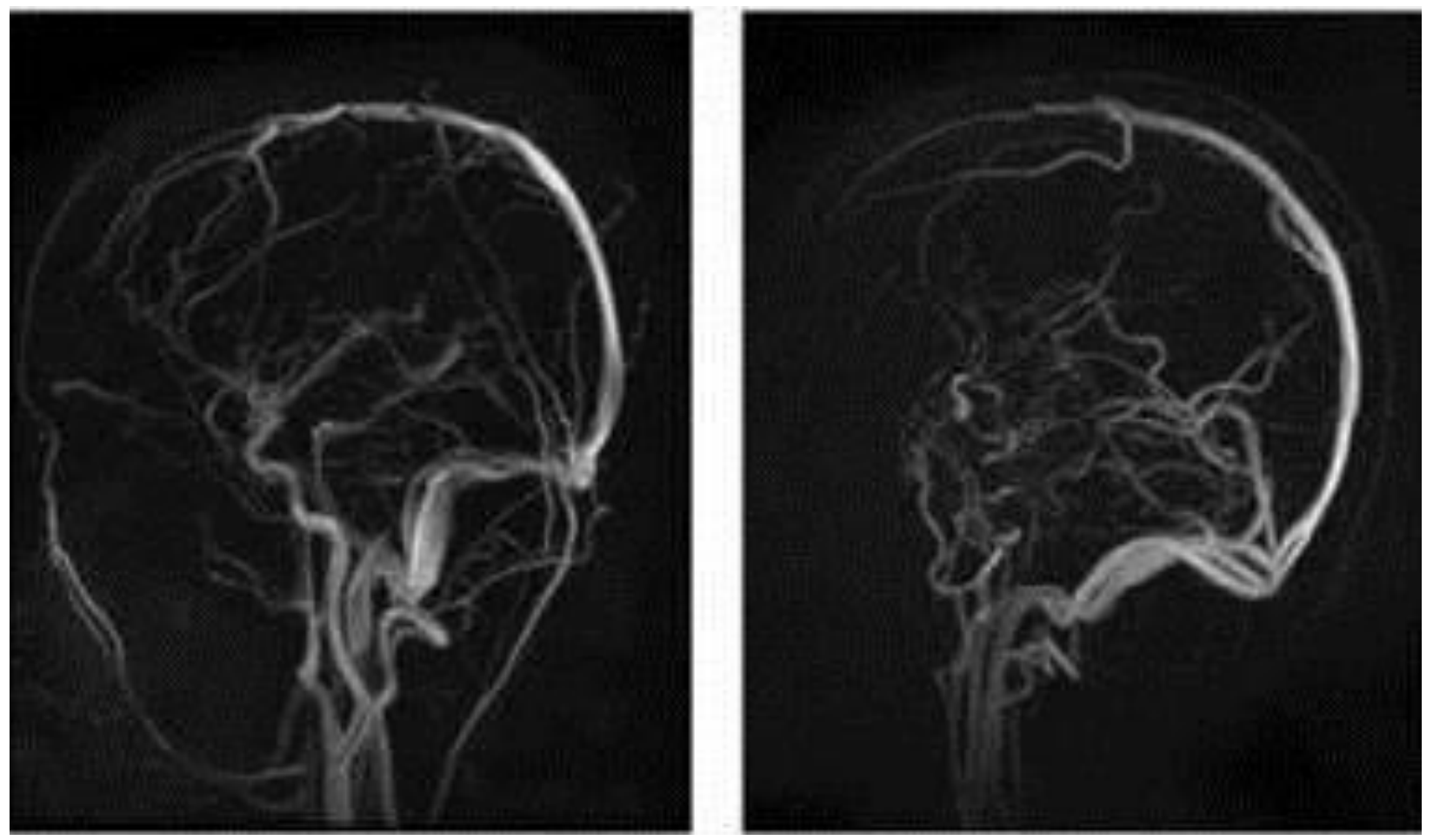

Figure 5. Hypoplasia of anterior $1 / 4$ and $1 / 2$ of superior sagittal sinus.

\section{Arachnoid granulations}

Arachnoid granulations are normal anatomical structures which protrude in to the dural sinuses. They allow CSF to escape into the venous system from the subarachnoid space. They may simulate cerebral venous thrombosis when they are large enough (Figure 8).

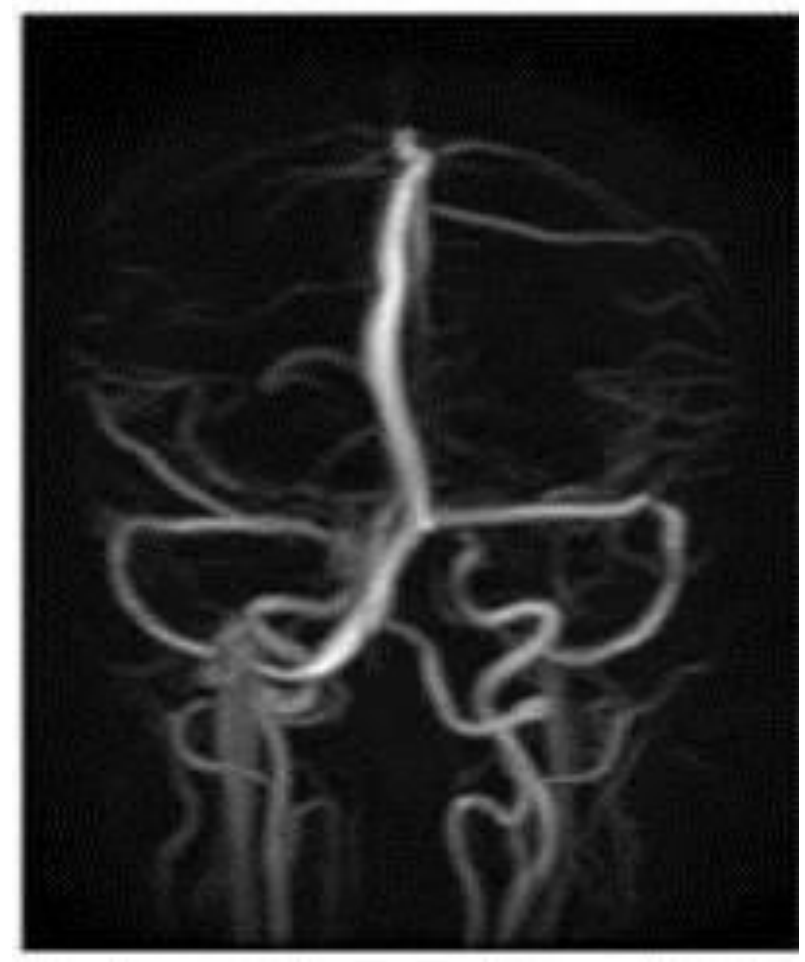

Figure 6. Right oblique sinus MRV 


\section{Pictorial essay $\mid$ SLJR}
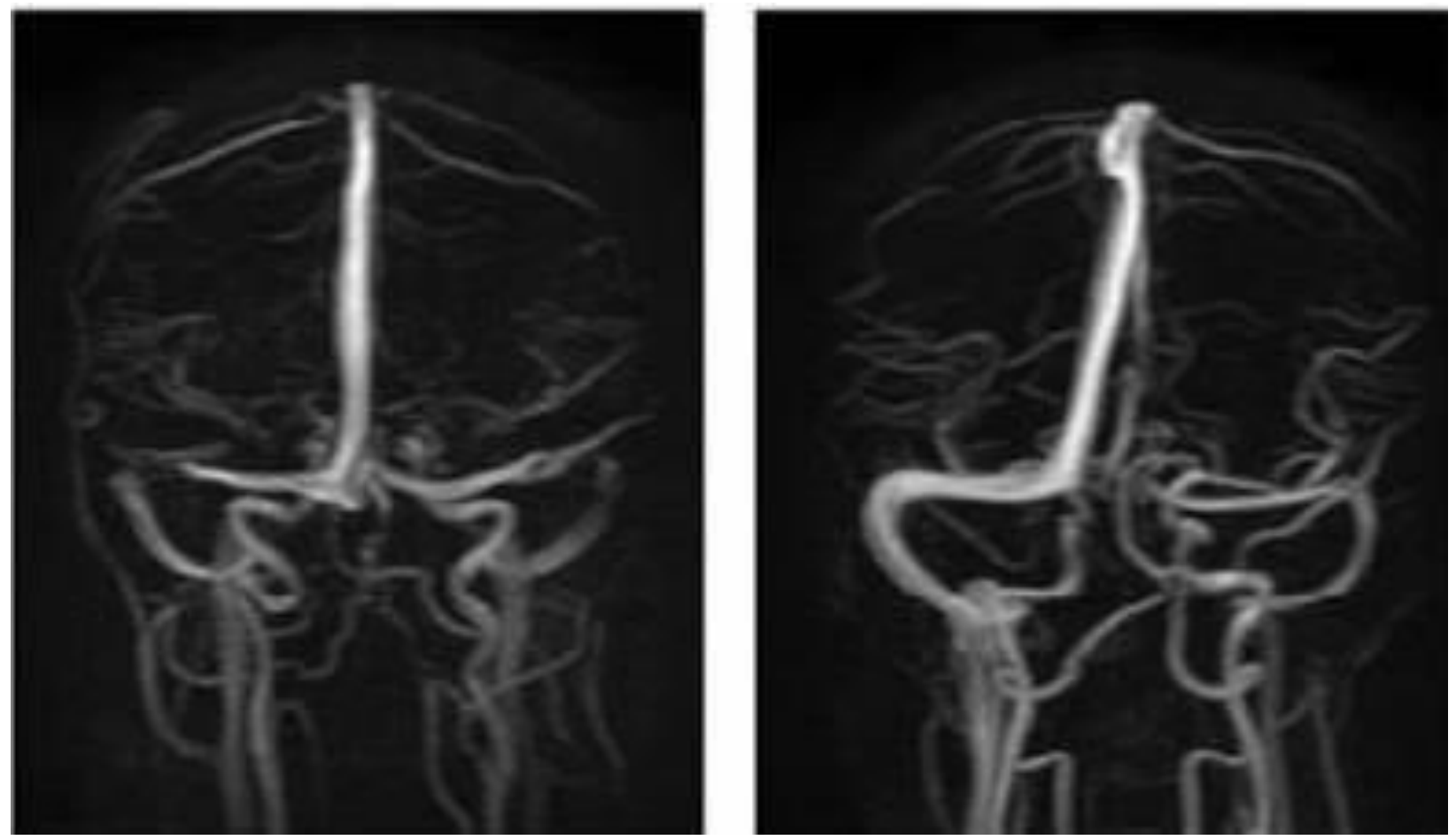

Figure 7. Flow gaps demonstrated in transverse sinuses MRV

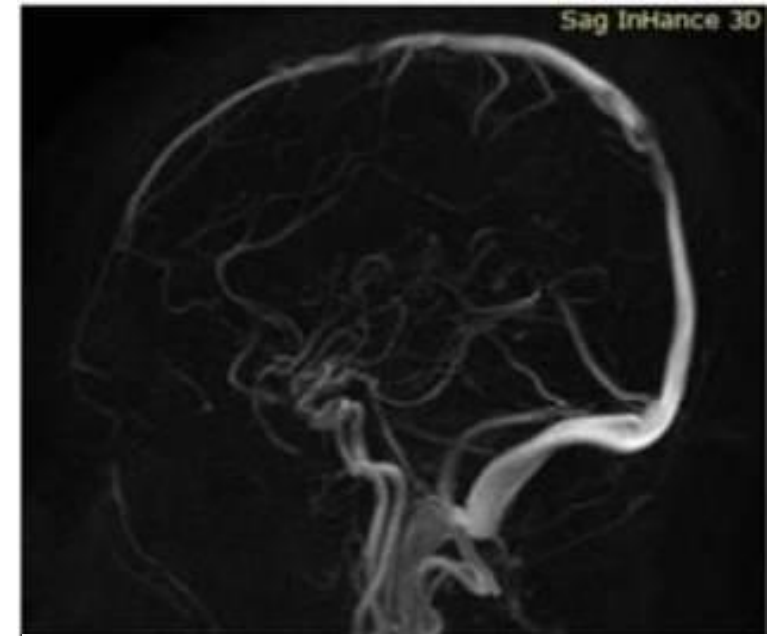

Figure 8. Arachnoid granulations seen as flow gaps in the superior sagittal sinus

\section{CONCLUSION}

As shown above, anatomic variants and artifacts occur often in non contrast enhanced MR venography. They tend to follow a constant pattern and their locations sometimes coincide with sites where
Cerebral Venous Sinus Thrombosis (CVST) is also common. These variants and artifacts can be potential pitfalls in the MRV diagnosis of CVST specially when there are no supportive imaging features such as brain infarcts or appropriate clinical background. Therefore, it is essential for radiologists to be familiar with their MRV characteristics and anatomy so that they are not misinterpreted as CVST.

(Authors wish to thank Mr. Indika Abeysinghe for performing MRV studies.)

Authors state that, some of the images and data contained in this pictorial essay are from the Authors' conference presentation

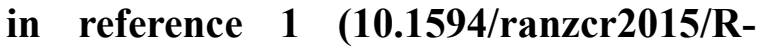
0005) 


\section{References}

1. Pallewatte AS, Liyanage N. Normal variations and artifacts in MR venography that may cause pitfalls in the diagnosis of cerebral venous sinus thrombosis. 10.1594/ranzcr2015/R0005 .

2. Zouaoui A, Hidden G. Cerebral venous sinuses: anatomical variants or thromboisis? Acta Anat (Basel). 1988;133:318-324.

3. Alper F, Kantarci M, Dane S, Gumustekin K, Onbas O, Durur I. Importance of anatomical asymmetries of transverse sinuses: an MR venographic study. Cerebro vasc Dis.2004;18(3):236-9.

4. R.H. Ayanzen, C. R.Bird, P.J.Keller, F. J. McCully, M.R.Theobald, J.E.Heiserman. Cerebral MR venography: Normal anatomy and potential diagnostic pitfalls. AJNR Am J Neuroradiol. 2000; 21:7478.

5. Rollins N, Ison C, Booth T, Chia J (2005) MR in the pediatric patient. AJNR Am J Neuroradiol. 26:50-55. 